\title{
Segmentation of Fruit and Vegetables using Fuzzy - Corner Based Region Growing Algorithm (Fuzzy-COBRA)
}

\author{
Mohana Maniganda Babu V, T.Santha
}

\begin{abstract}
This paper proposes a Novel algorithm called Fuzzy - Corner Based Region Growing Algorithm for shape analysis and object segmentation in Fruit image processing and specifically for Detection of Fruits and Vegetables in the large farms. The proposed research focus on extracting the corners of the object with varying shapes using the proposed Fuzzy-COBRA. It is used in corner and edges at the initial part and those corners are fuzzified and processed with region growing algorithm on input images. The process starts from an input image imported to system using imaging devices directly or benchmark Kaggle fruit Image Dataset and FIDS30 to detect the loss of information in the edges and corners of the imported edge due to noise and position offset. Proposed algorithm recognizes and removes false noise using surface filters and some acquisition tools to reach the accuracy level in detecting the shape of fruits, and vegetables. It solves the extreme points of the intersecting line of curved points. Proposed a Fuzzy-COBRA positions the corners of the imported image to capture important events and improves the object quality. The experimental results show the improve in efficiency to in segmenting the fruit and vegetables by detecting the corners after fuzzification and achieves higher accuracy in detecting the position of the edges from images of fruits and vegetables especially in organic agriculture during export and import in large scale.
\end{abstract}

Keywords : Fuzzy COBRA, Corner Detection, Edge, Surface Filters, Region Growing.

\section{INTRODUCTION}

Agricultural Image processing is a method to perform operations, analysis, and manipulation on an input image captured in an agricultural field using some acquisition tools and finally as an output it retrieves an enhanced characteristics or features associated with that input image in order to extract some useful information. In image processing, corner and edge detection plays a major role. In computer vision systems, corner detection approach helps in corner identification of the imported image. The combination of two or more edges results in formation of corners. It is used to extract some associated features on corners i.e. finding the corners of captured image or intersection points of two edges of input image and its applications are image registration like face recognition systems, video tracking and motion detection images. It is used to detect same corners of multiple input images. As a

Revised Manuscript Received on July 08, 2019.

Mohana Maniganda Babu , Research scholoar of Dr.G.R.D. College of Science, Coimbatore India.

Dr.Santha T, Principal of Dr.G.R.D. College of Science, Coimbatore India result, it improves the efficiency in finding the corners. Edge detection approach is to detect features of sharp edges in image brightness in order to capture changes in properties. It preserves the structural properties of an image and extracts the associated feature of the captured image. The Proposed algorithm works as an initial step by designing a test window with a threshold level. The captured or input image is passed into the designed test window. The window is encountered with a target test pixel and the image is rotated in all directions. As result of image window passing, an average energy in the window is measured or calculated. If the passed image value is higher than the threshold value, then the point is considered as a corner position of the image. If not, it is not accounted as a corner point.

\section{PROCEDURE FOR PAPER SUBMISSION}

Corner detection algorithm is used to detect, locate corners [1]. This is done to retrieve the associated features and certain identification using some acquisition tools [1]. Three steps are followed. The first step starts by using Barron operator to calculate the gradient of the image. Second step aims at smoothening the image hence to reduce and remove the noise by using a B-spline function. Third step to clarify whether the detected points are corners are not [2]. Since there might be chance of detecting irrelevant point as corner so constructing B-spline function excludes the neighboring points other than corner points [3]. Corner detection approach has been discussed [5]. Harris algorithm is used for corner detection for the captured image. It detects the corners of multiple images. [4]. Harris corner detection algorithm locates the important feature of image which contains useful information. It extracts the statistical features of standard deviation [5]. In this paper, corner detection is used for classifying breast mammograms as normal or abnormal. It is done with the help of the training data set used for Support Vector Machine (SVM). Here, improved Harris Corner Detection produces the output as corner pixels which are the taken as the input for the training set. [6]. In this paper, water marked region is taken as a captured or imported image [7]. The task is to detect the corner points of the input image. Harris corner detection algorithm is chosen to detect the corners of the water marked region. [8]. The result achieved improves the accuracy in tracking. In this research paper, the main focus is on localization of eyes in human faces. This is done with corner detection approach [9]. A new method of corner detection is analyzed. Experimental results show that the proposed algorithm works efficiently for

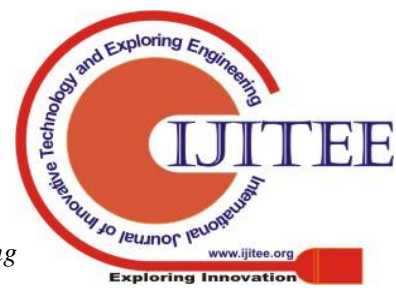


both grey scale and multi spectral images [10].

\section{EXISTING RESEARCH:}

\section{SURF}

Speeded Up Robust Features (SURF) is a newly developed framework, which is very likely to becoming the next fact feature detector in the industry. To improve performance of seeing system, there's got to address 2 issues: 1 st, there's got to emphasize the importance of the quantity of feature pairs once seeing is finished. Since this range is already gift within the recognition score, one potential improvement would be to boost its price to a bigger power. mistreatment this rule, it will generate a group of feature pairs between the question image and every individual info image. For seeing task, SURF rule is employed owing to its powerful attributes, together with scale invariability, translation invariability, lighting invariability, distinction invariability, and rotation invariability \& it will observe objects in pictures taken underneath totally different unessential and intrinsic settings. SURF approximates the DoG with box filters. rather than Gaussian averaging the image, rectangles are used for approximation since the convolution with square is far quicker if the integral image is employed. Also, this will be worn out parallel for various scales. The SURF uses a BLOB detector that relies on the boot matrix to search out the points of interest. For orientation assignment, it uses wave responses in each horizontal and vertical directions by applying adequate Gaussian weights. A neighbourhood round the key purpose is chosen and divided into subregions then for every subregion the wave responses are taken and depicted to induce SURF feature descriptor.

\section{PROPOSED METHODOLOGY}

The Proposed Research focus on extracting the features of Corners sieving. Divide the test image into 9 blocks and process each block independently to ensure that the detected corners are evenly distributed in the image without aggregation and thus eliminate the possibility that some corners may be lost due to the sharp contrast in gray level in different parts of the image, denote the corners detected in each block as set Corner [M], resort Corner [M]. At last, select the pixels of which $\mathrm{R}(\mathrm{x}, \mathrm{y})$ are relative large as the real corners. We use an adaptive way to select $[1 \times \mathrm{M}]$ from $\mathrm{M}$. The cyclic iterative method is used to ensure the value of 1 : select a little original value $1=0.02$ in $(0,1)$, and take $1=1+$ step to iterate circularly (set step 0.01 ). If $l=1$, end the iteration; otherwise judge that whether the block has any corner remained, end the iteration if it has. In addition, the corners in the border of adjacent blocks are abandoned.

\subsection{Fuzzy - Corner Based Region Growing Algorithm}

Our proposed Algorithm improves accuracy by removing false noise. Proposed algorithm detects the corners and edges using filters. It extracts the statistical features with maximum intensity and other associated features. The method used as follows,

1. Passing the captured image into a test window and decomposing the image using directional filter banks. Gradient calculation is applied to find out the grey level gradients

2. Our algorithm detects the corners in each direction using filters. Filters detect large redundancies. A $3 * 3$ B-Spline filter is used instead of Gaussian filter for smooth filtration with reduced noise. It retains corners with better and effective information

3. Filters detect the corner points by performing some mathematical formulas like standard deviation to extract the statistical features.

4. Detected corner points are combined by performing logical operations.

5. Dilation technique is applied to combine all aggregate corners into a single corner.

6. Identification of corner using centroid. The proposed algorithm reduces the false noise with better localization.

7. Corners are divided into 9 blocks. Each individual block is tested to ensure that detected corners are evenly distributed. Some corners are lost due to grey sharp contrast over different parts of images.

Efficiency and consistency of proposed algorithm is measured using the number of corner numbers found. It is denoted as CCN (Consistency of Corner Numbers). Consistency is defined as $\mathrm{CCN}=100 * 1.1-|\mathrm{Nt}-\mathrm{No}|$

8. All corner points are detected using our algorithm. And operator is used to extract the corner points common to R, G and B components. But this step does not detect whether the detected corners are false or true. Hence it follows an iterative method to detect the corner with false noise.

9. The iterative method is as follows. First the corner for original image is detected and measured using CCN. Next the original or captured image is rotated with an angle of 100 to detect the other possible corners. The rotation is targeted with a variation between -900 and +900 .

$\mathrm{CCN}=100 * 1.1-|\mathrm{Nt}-\mathrm{No}|$

No - number of corners detected in original image.

$\mathrm{Nt}$ - number of corners in the transformed image. Hence the efficiency is improved in our proposed algorithm compared to other existing algorithms.

10. Finally the corners in an image are detected. More corner points can be detected by changing the threshold values.

\section{RESULTS AND DISCUSSION}

Dataset: A 1000 fruits and vegetable images collected in agricultural farms of resolution $1024 \times 786$ resolution with minimum 800 dpi.

\section{Result:}

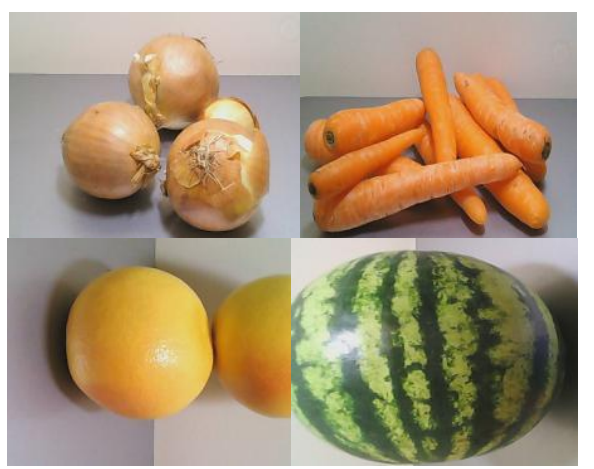


FIGURE A: Original Image - Left to Right

a. Onion b. Carrot c. Orange d. Watermelon

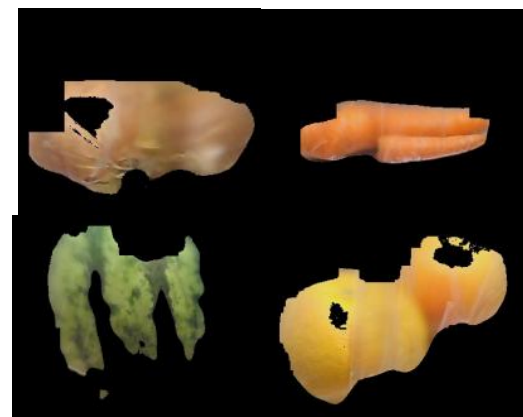

FIGURE B: Output Image - Left to Right

a. Onion b. Carrot c. Orange d. Watermelon

\section{Precision}

For finding the precision on a set of results, precision is the number of correct results divided by the number of all

$\pi=\mathrm{P}(\mathrm{f}(\mathrm{x})=\mathrm{C}+\| \mathrm{h} \theta(\mathrm{x})=\mathrm{C}+) \pi=\mathrm{P}(\mathrm{f}(\mathrm{x})=\mathrm{C}+\mid \mathrm{h} \theta(\mathrm{x})=\mathrm{C}+)$ given that we predict $\mathrm{xx}$ is + what's the probability that the decision is correct we estimate precision as $\mathrm{P}=\# \mathrm{TP} \#$ predicted positives = \# TP\# TP + \# FP.

Interpretation

- $\quad$ Out of all the fruit image, how many actually have decay?

- $\quad$ High precision is good

TABLE 1: Accuracy Table

we don't tell many fruits that they have decay when they actually don't

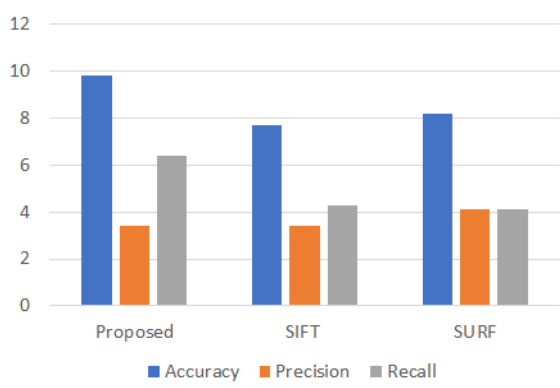

CHART 1: Accuracy Chart

Recall

Recall is the fraction of the relevant result that are successfully detected. $\rho=P(h \theta(x)=C+\| f(x)=C+) \rho=P(h \theta(x)=C+\mid f(x)=C+)$ given a positive instance $\mathrm{xx}$ what's the probability that we predict correctly we estimate recall as $\mathrm{R}=\# \mathrm{TP} \#$ actual positives $=\#$ $\mathrm{TP} \# \mathrm{TP}+\# \mathrm{FN} \mathrm{R}=\# \mathrm{TP} \#$ actual positives $=\# \mathrm{TP} \# \mathrm{TP}+\# \mathrm{FN}$ Interpretation returned results.

- $\quad$ Out of all the fruit image that do actually have disease, how much we identified? The higher the better. We don't fail to spot many fruits and vegetables that actually have diseases.

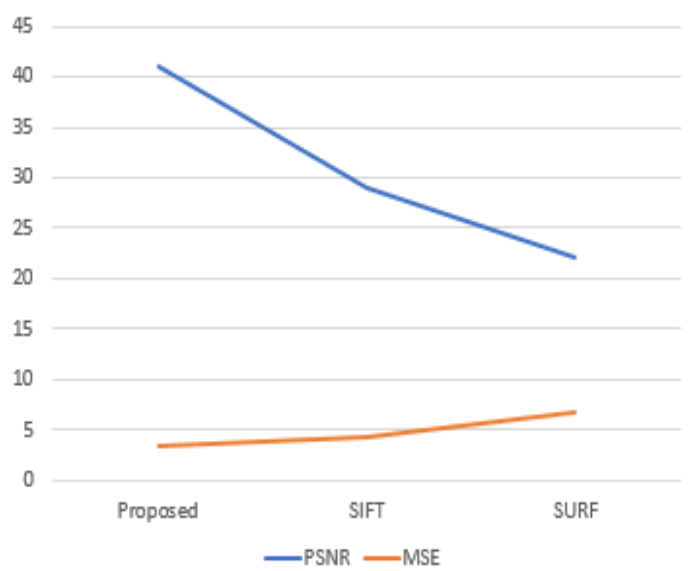

CHART 2: PSNR and MSE Chart

Accuracy

\begin{tabular}{|l|l|l|l|l|l|}
\hline $\begin{array}{l}\text { Met } \\
\text { hod }\end{array}$ & $\begin{array}{l}\text { Accu } \\
\text { racy }\end{array}$ & $\begin{array}{l}\text { Recal } \\
\mathbf{l}\end{array}$ & $\begin{array}{l}\text { Precisio } \\
\mathbf{n}\end{array}$ & $\begin{array}{l}\text { PSN } \\
\mathbf{R}\end{array}$ & MSE \\
\hline $\begin{array}{l}\text { Prop } \\
\text { osed }\end{array}$ & $\mathbf{9 . 8 6}$ & $\mathbf{3 . 4 3}$ & $\mathbf{6 . 4 2}$ & $\mathbf{4 1 . 3}$ & $\mathbf{3 . 4 5}$ \\
\hline SIFT & 7.72 & 3.46 & 4.35 & 29.8 & 4.27 \\
\hline $\begin{array}{l}\text { SUR } \\
\text { F }\end{array}$ & 8.23 & 4.15 & 4.18 & 22.1 & 6.73 \\
\hline
\end{tabular}

ccuracy rate is calculate d with the values of Recall and Precisio

n.

Accuracy $=$ \#TP + \#TN/ \#TOTAL

Keep your text and graphic files separate till when the text has been formatted and titled. don't use exhausting tabs, and limit use of onerous comes to just one return at the tip of a paragraph. don't add any reasonably page number anyplace within the paper. don't range text heads-the model can try this for you.

\section{Error Metrics}

Two of the error metrics accustomed compare the varied compression techniques area unit the Mean sq. Error (MSE) and also the Peak Signal to Noise magnitude relation (PSNR). The MSE is that the accumulative square error between the compressed and also the original image, whereas PSNR could be a live of the height error. The mathematical formulae for the 2 are

$$
\mathrm{MSE}=\frac{1}{\mathrm{MN}} \sum_{\mathrm{y}=1}^{\mathrm{M}} \sum_{\mathrm{x}=1}^{\mathrm{N}}\left[\mathrm{I}(\mathrm{x}, \mathrm{y})-\mathrm{I}^{\prime}(\mathrm{x}, \mathrm{y})\right]^{2}
$$

PSNR $=20 * \log 10(255 /$ sqrt $(\mathrm{MSE}))$

Where $\mathrm{I}(\mathrm{x}, \mathrm{y})$ is that the original image, $I^{\prime}(x, y)$ is that the approximated version (which is 
really the decompressed image) and $\mathrm{M}, \mathrm{N}$ area unit the scale of the pictures.

- A lower worth for MSE suggests that lesser error, and as seen from the inverse relation between the MSE and PSNR, this interprets to a high worth of PSNR.

Logically, the next worth of PSNR is sweet as a result of it implies that the magnitude relation of Signal to Noise is higher. Here, the 'signal' is that the original image, and also the 'noise' is that the error in reconstruction. So, if you discover a compression theme having a lower MSE (and a high PSNR), you'll be able to acknowledge that it's a higher one. By examination the opposite algorithms, we have a tendency to propose that Fruit Image Pattern Recognition rule as associate degree economical technique for detection the correct illness region, it's a high Accuracy Rate, High PSNR worth and extremely low Mean Error.

\section{Conclusion}

The proposed research focus on how the Fuzzy-COBRA algorithm segments the fruits and vegetables in an input or from an image which is captured and the corner features are extracted in Organic Fruits and Vegetables. Our proposed algorithm also works for fruit image dataset 30(FIDS30). This corner detection uses 0 or 1 fuzzy operator. This proposed algorithm improves the efficiency and consistency of segmentation of exact fruits and vegetables than existing method, it converts the input grey scale image into color and it increases the time complexity. Our proposed algorithm uses the above mentioned 0 and 1 operation for grey to image conversion of fruits, and vegetables.

\section{REFERENCES}

1. Yujing Qiao School of Mechanical and Power Engineering Harbin University of Science and Technology Harbin, China," Improved Harris Sub-pixel Comer Detection Algorithm for Chessboard Image,” 2013 2nd International Conference on Measurement, Information and Control

2. Tejendra Panchala,*, Hetal Patela, Ami Panchalb," License Plate Detection using Harris Corner and Character Segmentation by Integrated Approach from an Image," 7th International Conference on Communication, Computing and Virtualization 2016

3. Hyun I. Kim, Sung Shin, Wei Wang Department of Electrical Engineering and Computer Science, South Dakota State University, Brookings, SD 57006, USA" SVM-based Harris Corner Detection for Breast Mammogram Image Normal/Abnormal Classification"

4. G.A. Papakostas, E.D. Tsougenis, D.E. Koulouriotis, V.D. Tourassis,"On the Robustness of harris detector in image watermarking attacks," Optics Communications, Volume 284,Issue 91,1 September 2011,Pages 4394-4407

5. Yanshan Li1, Wei Shi1, Ailin Liu2 Affiliation1. ATR National Key Lab. of Defense Technology, Shenzhen University; Shenzhen 518060, China lys@szu.edu.cn Affiliation2. College of Information Engineering, Shenzhen University; Shenzhen 518060, China," A Harris Corner Detection Algorithm for Multispectral Images Based on the Correlation ," ICWMMN2015 Proceedings

6. Jiandong Su, Xiusheng Duan and Jing Xiao," Fast Detection Method of Checkerboard Corners Based on the Combination of Template Matching and Harris Operator," Third International Conference on Information Science and Technology March 23-25, 2013; Yangzhou, Jiangsu, China.

7. Nilanjan Dey, Poulami Das, Anamitra Bardhan Roy, Achintya Das," Detection and Measurement of Arc of Lumen Calcification from Intravascular Ultrasound using Harris Corner Detection", 2012 National Conference on Computing and Communication Systems (NCCCS) 978-1-4673-1953-9/12/\$31.00 @2012 IEEE

8. Na Yao, Tiecheng Bai, Xia Jiang, Haifang Lv, College of Information Engineering Tarim University Alaer, China," Improved Harris Corner
Detection for Chinese Characters," 2013 Fourth World Congress on Software Engineering.

9. M. Fatih Aydogdu, M. Fatih Demirci, and Cosku Kasnakoglu," Pipelining Harris Corner Detection with a Tiny FPGA for a Mobile Robot ," Proceeding of the IEEE International Conference on Robotics and Biomimetics (ROBIO)Shenzhen, China, December 2013

10. Yijian Pei, Hao Wu, Jiang Yu, Guanghui Cai School of Information Science and Engineering, Yunnan University, No. 2, North Green Lake Road Kunming, P.R.China, 650091," Effective Image Registration based on Improved Harris Corner Detection,” 2010 International Conference on Information, Networking and Automation (ICINA)

\section{AUTHORS PROFILE}

Mohana Maniganda Babu V Completed his M.Sc in Applied Science (Computer Technology) in VLB Janakiammal College of Engineering and Technology, Bharathiar University, Coimbatore, May 2003 . He is currently undergoing his Ph.D in Computer Science in Dr. G.R.D College of Science, Coimbatore, under the guidance of Dr.T Santha, Principal of Dr.G.R.D.College of Science. His area of interest is Digital Image Processing, Agricultural Image Processing and Computer Vision.

Dr.Santha T received her Post Graduation from P.S.G. College of Arts and Science, Coimbatore in 1987 and M.Phil. from Bharathiar University in 1991.She also completed her $\mathrm{PhD}$ in Computer Science from Bharathiar University during 2012. She is presently working as Principal of Dr.G.R.D. College of Science, Coimbatore. She has more than two decades of teaching and research experience. Her research centers on Computer Applications and Optimization Techniques, Multimodal Transportation, Computer Simulation, Digital Image Processing and Computer Vision. 